\title{
Rizotomia dorsal seletiva (RDS) para espasticidade: técnica minimamente invasiva no cone medular
}

\author{
Andre Kiss ${ }^{1}$, Tamara Rodrigues Pato Salles ${ }^{2}$, Haydee Cristina Freitas \\ de Morais ${ }^{3}$, Julio Aguiar Junior', Rafael Morais Kiss ${ }^{5}$
}

Clínica Neurológica de Joinville, SC, Brasil. Hospital Materno Infantil Dr. Jeser Amarante Faria, Joinville, SC, Brasil. Associação de Assistência à Criança Deficiente (AACD), Joinville, SC, Brasil.

\section{RESUMO}

A rizotomia dorsal seletiva é um procedimento cirúrgico bem aceito no manejo da espasticidade e tem como objetivo o alívio desse sintoma em pacientes cuidadosamente selecionados. Neste estudo, apresentamos o método que utilizamos em nosso serviço para indicação de pacientes, bem como a técnica cirúrgica e o método de monitorização neurofisiológica intraoperatória utilizado para otimizar os resultados. Realizamos também uma revisão bibliográfica utilizando os sites de busca da PubMed/ MedLine e Lilacs, com artigos publicados entre os anos de 1965 e 2013. Os descritores utilizados foram: "rizotomia", "espasticidade", "paralisia cerebral" e "monitorização intraoperatória". Foram selecionados 17 artigos para este estudo.

\section{PALAVRAS-CHAVE}

Rizotomia, espasticidade muscular, paralisia cerebral, monitorização intraoperatória.

\section{ABSTRACT}

Selective dorsal rhizotomy (SDR) for spasticity: minimally invasive technique at conus medullaris

Selective dorsal rhizotomy is a well-accepted cirurgic procedure utilized for relieving spasticity in carefully selected patients. In this work, we present the method we use to indicate the patients, the operative technique, and the intraoperative monitoring technique used in order to obtain the best results. We also reviewed the literature through the online databases PubMed/MedLine and Lilacs, with published papers from 1965 to 2013, descriptors included "rhizotomy", "spasticity", "cerebral palsy" and "intraoperative monitoring". We selected 17 articles for this study.

\section{KEYWORDS}

Rhizotomy, muscle spasticity, cerebral palsy, monitoring intraoperative.

1 Neurocirurgião da Clínica Neurológica e Neurocirúrgica de Joinville, do Hospital Materno Infantil Dr. Jeser Amarante Faria e da Associação de Assistência à Criança Deficiente (AACD), Joinville, SC, Brasil.

2 Médica fisiatra responsável pela Associação de Assistência à Criança Deficiente (AACD), Joinville, SC, Brasil.

3 Médica neurofisiologista da Clínica Neurológica de Joinville e do Neurocentro, Joinville, SC, Brasil.

4 Neurocirurgião do Hospital Municipal São José, Joinville, SC, Brasil.

5 Acadêmico de Medicina da Universidade Federal de Ciências da Saúde de Porto Alegre (UFCSPA), RS, Brasil. 


\section{Introdução}

A espasticidade é um dos distúrbios motores mais frequentes e incapacitantes observados em indivíduos com lesão do neurônio motor superior ao longo da via corticoespinhal. Muitos pacientes, especialmente as crianças com paralisia cerebral, têm um grau importante de espasticidade, que interfere na função motora e nas atividades de vida diária.

Seu quadro clínico é caracterizado pelo aumento do tônus muscular velocidade-dependente e pela exacerbação dos reflexos tendinosos profundos. É importante lembrar que a espasticidade pode ser agravada por vários fatores como infecções urinárias, úlceras de pressão, distensão visceral, imobilidade, lesões ungueais, alterações climáticas e fatores emocionais.

Existem, porém, fatores positivos relacionados à espasticidade como a compensação emocional da perda dos movimentos e a prevenção da atrofia muscular exagerada e da perda de massa óssea.

Dessa forma, o tratamento da espasticidade, quando necessário, deve ser multifatorial e interdisciplinar, pois necessita do controle de todos os fatores agravantes e da colaboração de vários profissionais como médicos, fisioterapeutas, terapeutas ocupacionais, psicólogos, entre outros.

Uma variedade de opções de tratamento para reduzir a espasticidade disfuncional tem sido desenvolvida, e encontrar o tratamento certo e no tempo certo para determinado paciente geralmente é um desafio. ${ }^{1}$

Uma opção bastante eficaz para a redução permanente da espasticidade em membros inferiores é a rizotomia dorsal seletiva (RDS).

A RDS é um procedimento cirúrgico definitivo, que secciona as raízes dorsais na região do cone medular, em determinados segmentos, visando diminuir a espasticidade. Como todo procedimento cirúrgico, é muito importante considerar os custos e os benefícios, em conjunto com o paciente e a família, de preferência com apoio psicológico, para procurar garantir uma expectativa adequada do conjunto familiar e a aderência aos cuidados e à reabilitação pós-operatória. Lembramos que é de suma importância que o paciente esteja inserido num ambiente de reabilitação.

Ao se avaliar o paciente para indicar o tratamento adequado, podemos utilizar várias escalas de avaliação. As mais utilizadas são:

- Escala Modificada de Ashworth; ${ }^{2}$

- Escala de Tardieu; ${ }^{3}$

- Escala de Avaliação de Automatismos Medulares de Lyon Université; ${ }^{4}$

- Escala de Reflexos Tendinosos; ${ }^{5}$

- Escalas de Funcionalidade:

- Índice de Barthel;:,7

- Medida de Independência Funcional. ${ }^{8}$

\section{Seleção de pacientes e indicação}

O objetivo funcional da RDS é a redução da hipertonia, proporcionando, assim, condições para melhora da força muscular e da função motora.

Revisões extensas sobre resultados da RDS mostram que vários resultados funcionais (mobilidade articular, força, marcha, posicionamento ao sentar) permanecem estáveis após vários anos. ${ }^{9}$ Também se verificam estabilização da subluxação do quadril e redução de procedimentos ortopédicos e de aplicações de toxina botulínica. ${ }^{10}$

Para obter o resultado desejado com a cirurgia, o grande foco de atenção do cirurgião deve ser a seleção dos pacientes. ${ }^{11}$

Um bom exame físico deve incluir os seguintes pontos:

- Observação da postura e posicionamento do paciente. Este dado, usualmente, traz consigo informações sobre o potencial motor do paciente. Deve-se atentar para controle cervical e de tronco e interação com o meio.

- Avaliação estática e dinâmica do tônus muscular e das amplitudes de movimento articular: observar a presença de encurtamentos, retrações musculares, deformidades ósseas, força muscular, espasticidade, distonia, atetose, coreia, tremores ou outras discinesias.

- Observação do desempenho em transferências e trocas posturais (deitar, rolar, sentar e ficar de pé). Apenas observando, é possível extrair importantes informações sobre os controles cervicais e de tronco, movimentações involuntárias, tônus muscular dinâmico, entre outros.

- Observação da marcha: solicitar ao paciente que caminhe com as órteses e meios auxiliares que utiliza e também sem esses equipamentos. Muitas vezes, a marcha desencadeia padrões espásticos específicos como a adução de coxas e pés equinos.

- Conversar com o paciente e também com o seu responsável. Isso ajudará a estimar um potencial cognitivo e de colaboração na reabilitação pós-cirúrgica, além de obter informações sobre de que forma a espasticidade está prejudicando seu desempenho em determinada função, o que lhe ajudará a estabelecer os objetivos da cirurgia.

Nesse contexto, é muito valioso dispor de uma equipe interdisciplinar, o que dará maior segurança à indicação cirúrgica. O neurocirurgião, o fisiatra, o ortopedista, o neuropediatra, o fisioterapeuta, o psicólogo e o assistente social são alguns profissionais-chave nesse processo. 
Os objetivos da cirurgia são individualizados, e o ideal é que sejam traçados em equipe. Os mais comuns são:

- Melhorar desempenho de marcha. Atenção especial deve ser voltada a esse objetivo, pois é muito comum que o paciente ou cuidador criem expectativa exagerada em relação à marcha, visando à cura da doença de base. É de suma importância que o cirurgião seja claro e explique seus objetivos para não haver frustação. Quando necessário, o psicólogo pode ajudar nas orientações pré-cirúrgicas.

- Minimizar riscos de deformidades ortopédicas futuras. Existem vários estudos que demonstram que a RDS diminui o número de cirurgias ortopédicas corretivas no futuro, bem como as torna menos invasivas quando ainda são necessárias.

- Facilitar uso de órteses de posicionamento para membros inferiores e superiores. A redução da espasticidade facilita o encaixe do membro às órteses, evitando lesões e auxiliando a independência do paciente para colocá-las e retirá-las.

- Facilitar ao cuidador a realização de higiene perineal e vestuário do paciente. Este item reflete de forma intensamente positiva na qualidade de vida do paciente e do cuidador, pois essas tarefas são realizadas várias vezes num mesmo dia.

- Melhorar o posicionamento do paciente na cadeira de rodas. Com isso, espera-se melhorar o conforto e diminuir a dor e o risco de escoliose e as complicações respiratórias associadas a ela.

A revisão da literatura aponta que os grupos que mais se beneficiam da RDS são pacientes com diparesia espástica, com bom controle cervical e de tronco e que apresentam potencial de marcha.

Nesses pacientes citados anteriormente, é possível visualizar e aferir melhor os benefícios, pois estes se refletem claramente na melhora do desempenho de marcha.

Existe também outro grupo, com indicação mais controversa. Nele se encontram os pacientes com tetraparesia sem potencial de marcha. Apesar de esses pacientes não apresentarem evolução para marcha, eles têm bons resultados quanto à melhora do posicionamento, do conforto, da higienização e do manuseio deles por seus cuidadores, o que é difícil de ser medido de forma fidedigna.

O grupo de pacientes com hemiparesia espástica também pode ser beneficiado com a rizotomia unilateral, objetivando-se a melhora da marcha.

Por não se descreverem bons resultados, recomenda-se excluir os pacientes com:

- Componente predominantemente distônico e atetótico;
- Doenças progressivas ou degenerativas.

O candidato ideal para a RDS, ou seja, aquele que mais pode se beneficiar com a cirurgia, é uma criança, com diparesia espástica, entre 4 e 6 anos de idade, que anda de forma independente, mas com padrão de marcha anormal. ${ }^{11}$ Ou seja, quanto melhor o nível de função motora pré-operatória, maiores serão os ganhos funcionais pós-operatórios. ${ }^{12}$

As complicações ocorrem em pequena porcentagem dos casos operados. ${ }^{13}$ As mais frequentemente relatadas na literatura são: broncoespasmo intraoperatório, pneumonia aspirativa, retenção urinária transitória, constipação, dores e disestesias em membros inferiores, também transitórias. Alguns pacientes podem apresentar também déficit sensitivo mais intenso (dor, temperatura e propriocepção), que tende a desaparecer nos meses seguintes.

\section{Procedimento cirúrgico}

A cirurgia tradicional é extensa e, em geral, envolve laminotomia osteoplástica de L1 a L5 com laminectomia de S1 e S2 como proposta por Peacock et al. ${ }^{14}$

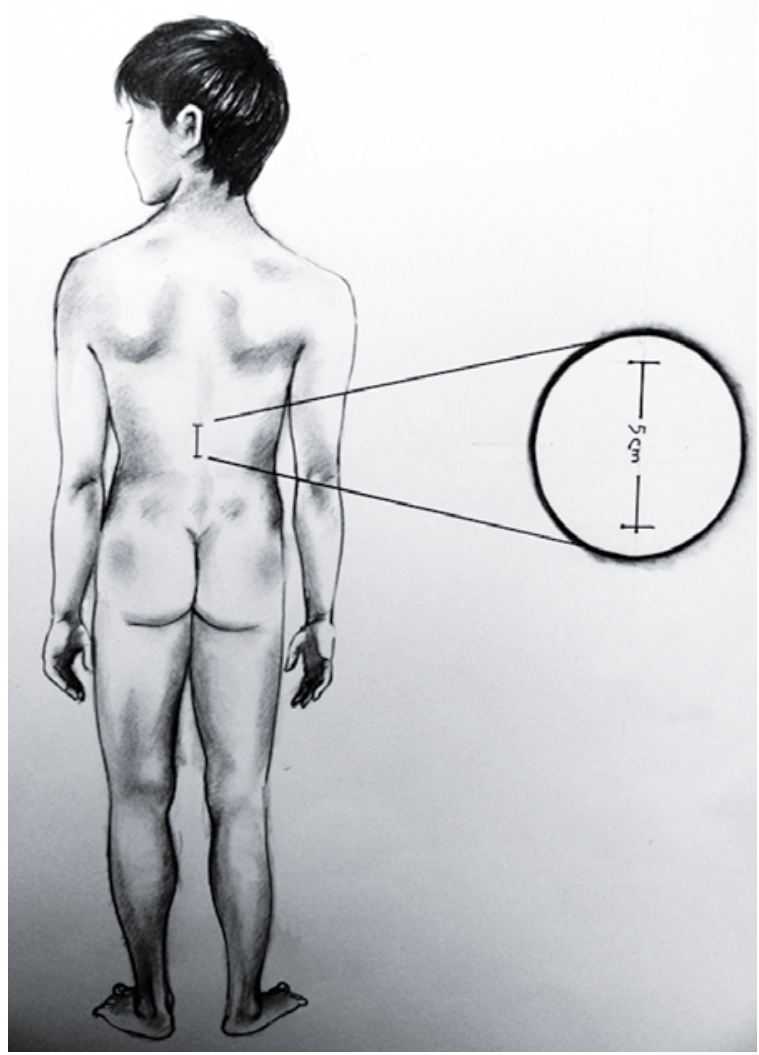

Figura 1 - Representação gráfica da incisão de aproximadamente $5 \mathrm{~cm}$ para o acesso ao cone medular. 
O procedimento cirúrgico que adotamos consiste em uma abordagem minimamente invasiva que envolve apenas a exposição do cone medular e do início da cauda equina (Figura 1), como descrito por Park e Johnston. ${ }^{15}$

Para essa abordagem mínima, é necessária a localização precisa do cone medular. Para isso realizamos, no pré-operatório, ressonância magnética $(\mathrm{RM}) \mathrm{da}$ região toracolombar, embora alguns autores utilizem ultrassonografia transoperatória para esse fim.

O paciente é colocado em decúbito ventral após a colocação dos eletrodos na musculatura dos membros inferiores e esfíncter anal. É realizada a localização radioscópica do corpo vertebral correspondente ao final do cone medular (previamente visualizado pela RM) e do forame neural de L1. A incisão, em geral de 5 centímetros, é então feita nesse nível.

Para exposição adequada do cone, fazemos a remoção completa de uma lâmina (geralmente L1) e de parte das lâminas superior e inferior, conforme a necessidade para boa exposição do cone.

Após a abertura dural e da aracnoide, não usamos irrigação com soro fisiológico para não interferir no registro neurofisiológico. Também evitamos ao máximo a coagulação de qualquer artéria adjacente às raízes ou ao cone.

Identificamos, então, a raiz L1 no seu forame correspondente. Em seguida, identificamos o final do cone, o filum terminal e as finas raízes sacrais (S3 a S5), que são deixadas intactas. Lateralmente a elas, temos S2, que é mais volumosa.

Com uma leve angulação lateral do microscópio, conseguimos visualizar facilmente a zona de entrada das raízes dorsais e sua separação em relação às raízes ventrais. Após dissecção cuidadosa, inserimos pequena tira feita de luva com 5 milímetros de largura separando as raízes ventrais das dorsais de S2 a L2. Para confirmação de que não incluímos nenhuma raiz ventral, nesse passo realizamos estímulo mecânico com o dissector (tap test), ou utilizamos estimulação elétrica de baixa intensidade, e observamos se ocorre resposta muscular pela eletromiografia (EMG).

Em seguida, realizamos a separação individual das raízes usando alguns reparos anatômicos e dissecção meticulosa: L2 dirigindo-se para o seu forame vertebral, S2 como fascículo único, lateralmente às finas raízes de S3-S5. A separação precisa das raízes às vezes não é possível, porém isso não é crítico para a realização da RDS por esse método, já que nesses pacientes a inervação motora provém de vários segmentos.

Cada raiz é subdividida, então, em três a cinco partes, que são suspensas por eletrodos (Figura 2) e testadas separadamente: as radículas com respostas anormais (graus 3 e 4 ) são seccionadas, tentando se preservar ao menos $1 / 3$ de cada raiz posterior. $O$ processo é realiza-

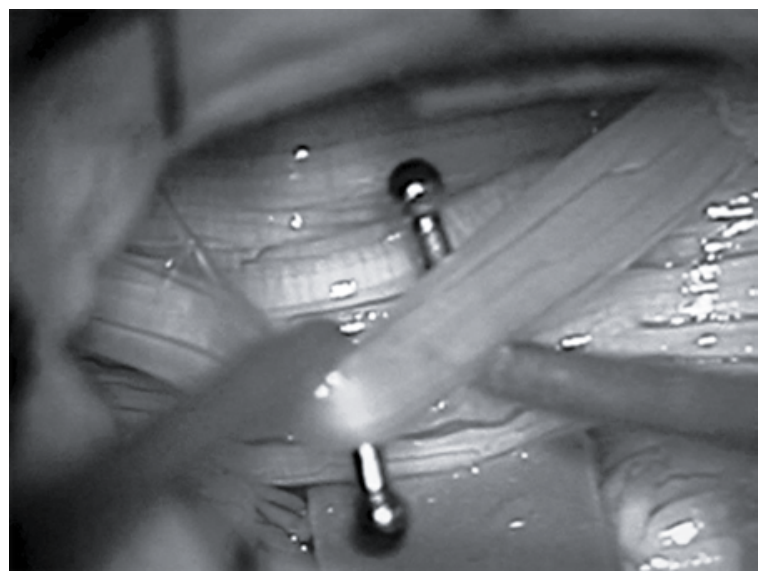

Figura 2 - Elevação de raiz dorsal com eletrodos em " $L$ ".

do dessa forma de L2 a S2 bilateralmente. Em seguida, realizamos a secção de $50 \%$ da raiz dorsal de L1, sem o estudo eletromiográfico, para redução da hipertonia nos flexores do quadril. ${ }^{15}$

\section{Avaliação neurofisiológica intraoperatória}

O objetivo da avaliação neurofisiológica durante a cirurgia é propiciar a seleção das radículas sensitivas com as respostas mais anormais para secção cirúrgica.

A rizotomia dorsal seletiva (RDS), baseada em critérios neurofisiológicos, vem sendo cada vez mais utilizada, com resultados bastante favoráveis.

Durante o procedimento intraoperatório, são realizadas estimulações das divisões das raízes posteriores, e as respostas são avaliadas e graduadas.

Essa avaliação permite ao neurocirurgião seccionar as divisões radiculares mais envolvidas na espasticidade, otimizando o resultado da cirurgia, com melhora da função e mínimas alterações sensitivas e motoras. ${ }^{16}$

A seleção das subdivisões das raízes é baseada na resposta eletromiográfica à estimulação elétrica (eletromiografia estimulada), seguindo-se critérios que serão mais bem descritos posteriormente. Os registros são realizados por meio de eletrodos de agulha de $2,5 \mathrm{~cm}$, colocados em pares, no esfíncter anal e em mais cinco grupos musculares dos membros inferiores, a seguir: adutores do quadril, quadríceps, tibial anterior, isquiotibiais e gastrocnêmio. ${ }^{17}$

Durante a estimulação, dois eletrodos especialmente desenhados para rizotomia (com terminações em forma de gancho, para permitir o isolamento da divisão radicular que se deseja estimular) são posicionados com uma distância de 5 a $10 \mathrm{~mm}$ entre eles. A intensidade do estímulo vai variando durante o procedimento, mas 
tipicamente são necessários entre 10 e $100 \mathrm{mV}$, com duração de $0,1 \mathrm{~ms}$.

O primeiro passo da avaliação é determinar o limiar (threshold) do músculo inervado pela subdivisão (rootlet) a ser avaliada.

Após o neurocirurgião dividir cada raiz posterior em três a quatro subdivisões, estas são estimuladas separadamente, com pulsos isolados $(0,5 \mathrm{~Hz})$. As respostas são captadas pelos eletrodos localizados nos diversos músculos e registradas na tela do equipamento.

Gradualmente, a intensidade do estímulo é aumentada, até o limiar ser atingido (menor nível de corrente elétrica necessária para produzir uma resposta no músculo). Em seguida, estimulação tetânica (train), com frequência de $50 \mathrm{~Hz}$, com a mesma intensidade do limiar que foi estabelecido previamente, é aplicada durante 1 segundo na mesma subdivisão.

A tela de registro é configurada com uma varredura de $4 \mathrm{~ms}$ /divisão para obtenção do limiar e, depois, de $200 \mathrm{~ms} /$ divisão para a estimulação tetânica $(50 \mathrm{~Hz})$. A sensibilidade (amplitude) é mantida em 200 uV/divisão durante ambas as estimulações.

Diversos tipos de respostas têm sido vistos durante essas estimulações tetânicas, entre eles respostas quadradas, em decremento, em incremento e sustentada.
As respostas são interpretadas de acordo com a distribuição delas nos vários grupos musculares e, ainda, se estão presentes unilateral ou bilateralmente. A tabela 1 demonstra as graduações das respostas tetânicas.

As divisões que produzem uma resposta “0" são deixadas intactas. As que produzem respostas $\underline{3+}$ e $\underline{4+}$ são seccionadas e aquelas com respostas $\underline{1+\mathrm{e}} \underline{2}+$ são geralmente poupadas. Se durante a avaliação apenas as respostas $\underline{1+}$ e $\underline{2+}$ são detectadas, escolhemos entre

\begin{tabular}{|c|c|}
\hline & $\begin{array}{l}\text { Tabela } 1 \text { - Critérios para graduar as } \\
\text { respostas eletromiográficas em RDS }\end{array}$ \\
\hline Grau & Resposta EMG \\
\hline 0 & $\begin{array}{l}\text { Não sustentada ou resposta simples após } \\
\text { estimulação tetânica. Resposta normal }\end{array}$ \\
\hline $1+$ & $\begin{array}{l}\text { Resposta sustentada em músculos inervados por meio } \\
\text { dos segmentos estimulados na extremidade ipsilateral }\end{array}$ \\
\hline $2+$ & $\begin{array}{l}\text { Resposta sustentada em músculos inervados por } \\
\text { meio dos segmentos estimulados e em músculos } \\
\text { de segmentos imediatamente adjacentes }\end{array}$ \\
\hline $3+$ & $\begin{array}{l}\text { Resposta sustentada em músculos inervados por meio } \\
\text { dos segmentos estimulados, assim como em músculos } \\
\text { inervados por segmentos mais distantes do estimulado }\end{array}$ \\
\hline $4+$ & $\begin{array}{l}\text { Resposta sustentada em múltiplos segmentos } \\
\text { do membro ipsilateral, atingindo também } \\
\text { músculos do membro contralateral }\end{array}$ \\
\hline
\end{tabular}
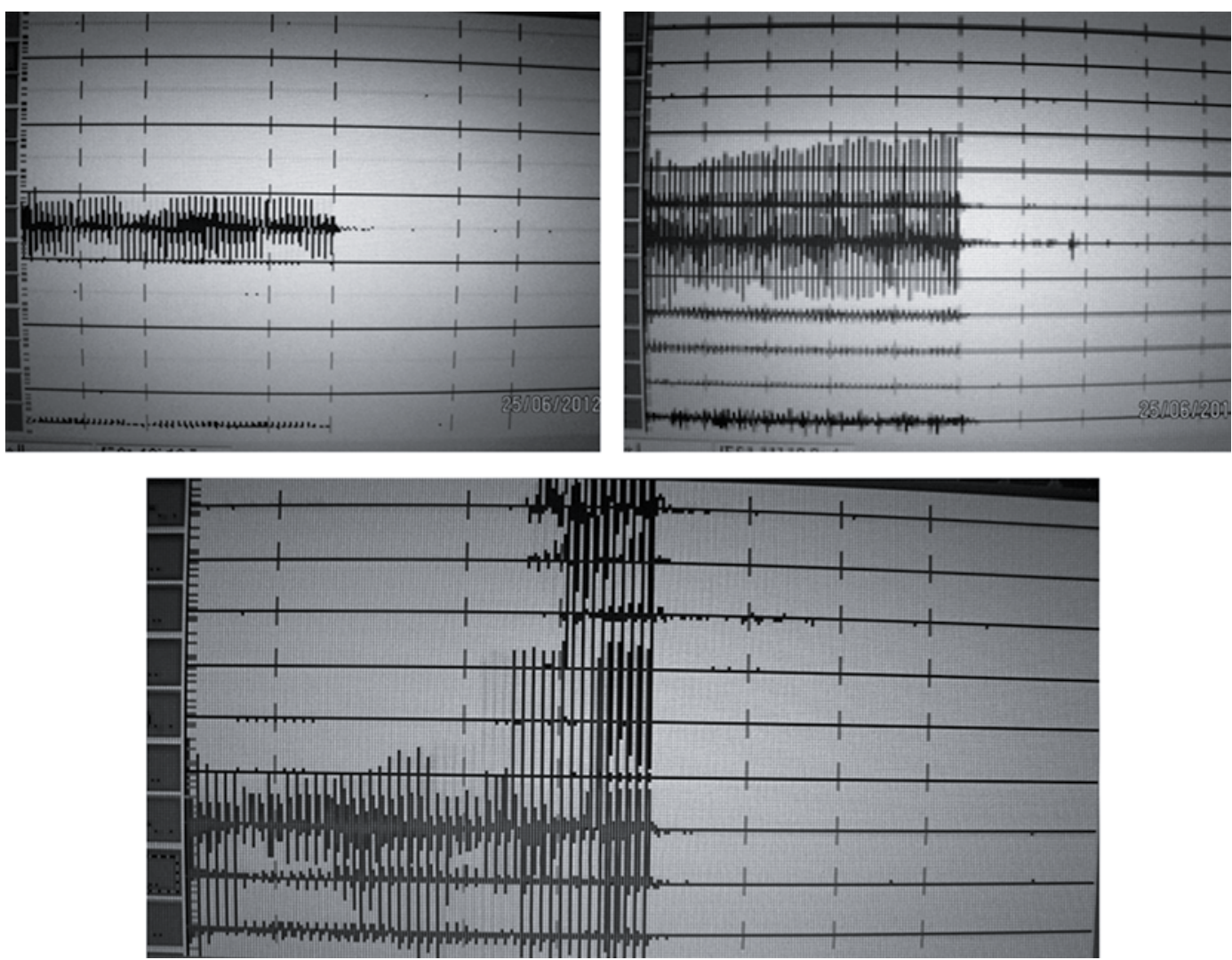

Figura 3 - No sentido horário, exemplos de respostas grau 1+, grau 2+e grau 4+ (traçados da metade inferior correspondem ao lado direito e da metade superior, ao lado esquerdo). 
essas respostas as divisões com as respostas mais ativas para serem seccionadas (Figura 3).

Em geral, entre $25 \%$ e $50 \%$ das divisões das raízes são seccionadas (17). A nível sacral, particularmente em S2, qualquer atividade a nível do esfíncter anal é uma contraindicação à secção dessa raiz, mesmo se houver respostas anormais nas extremidades.

Durante a rizotomia dorsal seletiva, a decisão de seccionar ou poupar as divisões das raízes posteriores é baseada primariamente no padrão das respostas eletromiográficas após a estimulação tetânica, porém alguns aspectos clínicos também devem ser considerados (distribuição e severidade da espasticidade), assim como o grau funcional do paciente.

\section{Considerações finais}

A RDS é uma ferramenta importante para redução da espasticidade e melhora da função motora em crianças, principalmente quando secundária à paralisia cerebral. Deve ser o passo inicial no tratamento cirúrgico desses pacientes, seguido de um programa abrangente de reabilitação e procedimentos ortopédicos.

A equipe interdisciplinar é um grande diferencial na condução dos casos, facilitando a seleção dos pacientes para as cirurgias, o delineamento dos objetivos, o preparo pré-operatório e o tratamento pós-operatório.

A cirurgia minimamente invasiva no cone elimina as desvantagens da cirurgia tradicional (laminectomia ou laminotomia osteoplástica de L1 a S1), tais como incisão longa, extensa dissecção muscular, secção de múltiplas lâminas, risco de lesão das raízes ventrais durante a dissecção das raízes dorsais, dor pós-operatória significativa.

Evolutivamente, observa-se menor incidência de dor lombar e deformidades espinhais com a cirurgia no nível do cone medular.

\section{Conflito de interesses}

Os autores declaram não haver conflito de interesses.

\section{Referências}

1. Nordmark E, Josenby AL, Lagergren J, Andersson G, Strömblad LG, Westbom L. Long-term outcomes five years after selective dorsal rhizotomy. BMC Pediatr. 2008;8:54.

2. Bohannon RW, Smith MB. Interrater reliability of a modified Ashworth scale of muscle spasticity. Phys Ther. 1987;67(2):206-7.

3. Graham HK. Botulinum toxin type a management of spasticity in the context of orthopaedic surgery for children with spastic cerebral palsy. Eur J Neurol. 2001;8(Suppl 5):30-9.

4. Millet MF, Mortamais J, Sindou M, Eyssette M, Boisson D, Bourret J. Neurochirurgie dans le paraolegic spastiques. Resultats a moyen treme de la radicellotomie posterieure selective. In: Simon L. Actualites en reeducation fonctionnelle et readaption. Paris: Masson; 1981. p. 76-85.

5. Meythaler JM. Concept of spastic hypertonia. Phys Med Rehabil Clin N Am. 2001;12(4):725-32.

6. Mahoney FI, Barthel DW. Functional evaluation. The Barthel Index. Md State Med J. 1965;14:61-5.

7. Lianza S, Koda LC. Avaliação clínica da incapacidade. In: Lianza S. Medicina de reabilitação. $3^{\mathrm{a}}$ ed. Rio de Janeiro: Guanabara Koogan; 2001. p. 11-21.

8. Lianza S, Koda LC. Avaliação da capacidade. In: Lianza S. Medicina de reabilitação. $3^{\mathrm{a}}$ ed. Rio de Janeiro: Guanabara Koogan; 2001. p. 296.

9. Mittal S, Farmer JP, Al-Atassi B, Gibis J, Kennedy E, Galli C, et al. Long-term functional outcome after selective posterior rhizotomy. J Neurosurg. 2002;97(2):315-25.

10. Hurvitz EA, Marciniak CM, Daunter AK, Haapala HJ, Stibb SM, McCormick SF, et al. Functional outcomes of childhood dorsal rhizotomy in adults and adolescents with cerebral palsy. J Neurosurg Pediatr. 2013;11(4):380-8.

11. Drazin D, Auguste K. Contemporary dorsal rhizotomy surgery for the treatment of spasticity in childhood. In: Quinones-Hinojosa A, editor. Schmidek \& Sweet operative neurosurgical techniques: indications, methods and results. Philadelphia: Elsevier/Saunders; 2012. p. 753-8.

12. Farmer JP, Sabbagh AJ. Selective dorsal rhizotomies in the treatment of spasticity related to cerebral palsy. Childs Nerv Syst. 2007;23(9):991-1002.

13. Abbott R. Complications with selective posterior rhizotomy. Pediatr Neurosurg. 1992;18(1):43-7.

14. Peacock WJ, Arens LJ, Berman B. Cerebral palsy spasticity. Selective posterior rhizotomy. Pediatr Neurosci. 1987;13(2):61-6.

15. Park TS, Johnston JM. Surgical techniques of selective dorsal rhizotomy for spastic cerebral palsy. Technical note. Neurosurg Focus. 2006;21(2):e7.

16. Steinbok P, Keyes R, Langill L, Cochrane DD. The validity of electrophysiological criteria used in selective functional posterior rhizotomy for treatment of spastic cerebral palsy. J Neurosurg. 1994;81(3):354-61.

17. Zakhary R, Smyth M, Peacock WJ. Dorsal rhizotomy for spasticity. In: Gandhi CD, Schulder M, editors. Handbook of stereotactic and functional neurosurgery. New York: CRC Press; 2003. p. 487-500.

Endereço para correspondência

Andre Kiss

Rua Ex-Combatentes, 125, Saguacu

89221-103 - Joinville, SC, Brasil

Telefone: (47) 9974-6487

E-mail: andrekiss@uol.com.br 